Louisiana State University

LSU Digital Commons

Faculty Publications

Department of Mathematics

$1-18-2018$

\title{
Bounded backstepping through a dynamic extension with delay
}

Frederic Mazenc

Laboratoire des Signaux et Systèmes

Michael Malisoff

Louisiana State University

Laurent Burlion

ONERA Centre de Toulouse

Victor Gibert

Airbus France

Follow this and additional works at: https://digitalcommons.Isu.edu/mathematics_pubs

\section{Recommended Citation}

Mazenc, F., Malisoff, M., Burlion, L., \& Gibert, V. (2018). Bounded backstepping through a dynamic extension with delay. 2017 IEEE 56th Annual Conference on Decision and Control, CDC 2017, 2018-January, 607-611. https://doi.org/10.1109/CDC.2017.8263727

This Conference Proceeding is brought to you for free and open access by the Department of Mathematics at LSU Digital Commons. It has been accepted for inclusion in Faculty Publications by an authorized administrator of LSU Digital Commons. For more information, please contact ir@lsu.edu. 
archives-ouvertes

\section{Bounded Backstepping through a Dynamic Extension with Delay}

Frédéric Mazenc, Michael Malisoff, Laurent Burlion, Victor Gibert

\section{To cite this version:}

Frédéric Mazenc, Michael Malisoff, Laurent Burlion, Victor Gibert. Bounded Backstepping through a Dynamic Extension with Delay. 56th IEEE Conference on Decision and Control (CDC 2017), Dec 2017, Melbourne, Australia. pp.607-611, 10.1109/cdc.2017.8263727 . hal-01660133

\section{HAL Id: hal-01660133 \\ https://hal.inria.fr/hal-01660133}

Submitted on 10 Dec 2017

HAL is a multi-disciplinary open access archive for the deposit and dissemination of scientific research documents, whether they are published or not. The documents may come from teaching and research institutions in France or abroad, or from public or private research centers.
L'archive ouverte pluridisciplinaire HAL, est destinée au dépôt et à la diffusion de documents scientifiques de niveau recherche, publiés ou non, émanant des établissements d'enseignement et de recherche français ou étrangers, des laboratoires publics ou privés. 


\section{Bounded Backstepping through a Dynamic Extension with Delay}

Frederic Mazenc
Michael Malisoff
Laurent Burlion
Victor Gibert

\begin{abstract}
We provide a bounded backstepping result that ensures global asymptotic convergence for a broad class of partially linear systems with an arbitrarily large number of integrators. We use one artificial delay, and we assume that the nonlinear subsystems satisfy a converging-input-convergingstate assumption. When the nonlinear subsystem is control affine with the state of the first integrator as the control, we provide sufficient conditions for our converging-inputconverging-state assumption to hold. Our example illustrates the novelty and utility of our main result.
\end{abstract}

Index Terms - Backstepping, delays, stabilization

\section{INTRODUCTION}

This work continues our team's search (begun in [7], [8], [9], [10], [12], and [13]) for new backstepping results that can help overcome the obstacles to using standard backstepping; see [5] for traditional backstepping. Classical backstepping synthesizes globally asymptotically stabilizing feedbacks, by recursively finding globally asymptotically stabilizing controls and Lyapunov functions for subsystems; see [6] for more recent backstepping that includes nonlinearities and uncertainties, and see [1], [2], and [3] for applications of backstepping for adaptive, aerospace, and robotic systems. However, there are important cases that call for backstepping where existing backstepping methods do not apply, e.g., for general nonlinear subsystems having bounds on the allowable control magnitudes, which produce challenges that we help address here.

We focus on systems having the form

$$
\left\{\begin{aligned}
\dot{x}(t) & =\mathcal{F}\left(t, x(t), z_{1}(t)\right) \\
\dot{z}_{i}(t) & =z_{i+1}(t), i \in\{1, \ldots, k-1\} \\
\dot{z}_{k}(t) & =u(t)+\sum_{j=1}^{k} v_{j} z_{j}(t)
\end{aligned}\right.
$$

with a scalar control $u$ and any number $k \geq 2$ of integrators. Here, $x$ is valued in $\mathbb{R}^{n}$ for any $n$, the $v_{j}$ 's are constant real numbers, and the nonlinear subsystem will enjoy a converging-input-converging-state condition that we define and discuss below. We write our closed loop controls as $u(t)$, but they will be feedbacks that depend on $t$ through their dependence on states of an enlarged system.

Mazenc is with EPI DISCO INRIA-Saclay, Laboratoire des Signaux et Systèmes (L2S, UMR CNRS 8506), CNRS, CentraleSupélec, Université Paris-Sud, 3 rue Joliot Curie, 91192, Gif-sur-Yvette, France, Frederic.MAZENC@1ss.supelec.fr.

Malisoff is with the Department of Mathematics, Louisiana State University, Baton Rouge, LA 70803-4918, USA, malisoff@lsu.edu.

Burlion is with ONERA, The French Aerospace Lab, 31055 Toulouse, France, lburlion@onera.fr

Gibert is with Airbus Operations, S.A.S, Toulouse, France. victor.gibert@airbus.com.

Malisoff was supported by NSF Grant 1408295.
While we assume that the current state of the system can be measured, we also use a delay in the state values in our feedback since this artificial delay is needed to design our bounded control. Our work [10] used the converginginput-converging-state assumption and an artificial delay, but a significant improvement in this note in comparison with [10] is that here we allow an arbitrarily large number $k$ of integrators, while [10] only allowed one integrator. Our bounded backstepping work [12] also allowed an arbitrarily large number of integrators, but the present work produces a globally bounded control for (1) while the controls for the original systems in [12] were not bounded. In addition, while [12] required $k$ artificial delays and did not use dynamic extensions, here we only require one artificial delay, which may make our feedback simpler to apply.

Our works [7], [8], [9], and [13] did not use converginginput-converging-state conditions or artificial delays. Moreover, our work differs from [8] (which was based on a forwarding method for one integrator case), [7] (which was limited to one integrator), [13] (which gives unbounded controls), and [16] and [17] (which employ Lie derivatives without satisfying input constraints). Hence, our novel combination of converging-input-converging-state conditions, artificial delays, and bounded controllers for (1) in this work is useful.

We use standard notation and definitions. We omit arguments of functions when they are clear, and the dimensions of our Euclidean spaces are arbitrary. We use $|\cdot|$ to denote the usual Euclidean norm and the induced matrix norm, and $|\phi|_{\infty}$ (resp., $|\phi|_{\mathcal{I}}$ ) is the essential supremum (resp., supremum over any interval $\mathcal{I}$ ) for bounded measurable functions; $\phi$. Set $\mathbb{N}=\{1,2, \ldots\}$. For a fixed constant $T>0$, let $C_{\text {in }}$ denote the set of all continuous functions $\phi:[-T, 0] \rightarrow \mathbb{R}^{a}$, which we call the set of all initial functions. We define $\Xi_{t} \in C_{\text {in }}$ and $\dot{\Xi}_{t} \in C_{\text {in }}$ by $\Xi_{t}(s)=\Xi(t+s)$ and $\dot{\Xi}_{t}(s)=\Xi^{\prime}(t+s)$ for all choices of $\Xi, s \leq 0$, and $t \geq 0$ for which the equalities are defined. We assume for simplicity that the initial times for our solutions are $t_{0}=0$ and that the initial functions are constant at time 0 (so the states are constant on $[-T, 0]$, where $T$ will be the artificial delay). We denote by $f^{(i)}(t)$ the $i$ th derivative of a function $f:[0,+\infty) \rightarrow \mathbb{R}$ with $f^{(0)}(t)=f(t)$, and $\sigma_{r}: \mathbb{R} \rightarrow[-r, r]$ is the standard saturation that is defined for all constants $r>0$ by $\sigma_{r}(s)=s$ for all $s \in[-r, r]$ and $\sigma_{r}(s)=r \operatorname{sign}(s)$ otherwise.

\section{MAIN RESUlt}

To state our theorem, we require the following two lemmas, the first of which follows from elementary calculations that we omit: 
Lemma 1: Let $q>0$ and $T>0$ be given constants, and $\mu_{0}:[-T,+\infty) \rightarrow \mathbb{R}$ be any continuous function, and set

$$
\begin{aligned}
& \zeta(t)=\int_{t-T}^{t} e^{q(\ell-t)} Q(t, \ell, \ell+T) \mu_{0}(\ell) \mathrm{d} \ell, \\
& \text { and } \Omega_{j}(t)=\zeta^{(j-1)}(t) \\
& \text { and } \mu_{i}(t)=\frac{1}{T} \int_{t-T}^{t} e^{q(\ell-t)} \frac{(t-\ell)^{i-1}}{(i-1) !} \mu_{0}(\ell) \mathrm{d} \ell
\end{aligned}
$$

for all $j \in\{1, \ldots, k+1\}$ and $i \in \mathbb{N}$, where $Q(t, a, b)=$ $(t-a)^{k-1}(t-b)^{k-1}$ for all $a \in \mathbb{R}$ and $b \in \mathbb{R}$ and $k \in \mathbb{N}$ with $k \geq 2$. Then there are constants $c_{i, j}(q, T) \in \mathbb{R}$ for all $i \in\{1, \ldots, 2 k-1\}$ and all $j \in\{1, \ldots, k\}$, and constants $g_{i}(q, T) \in \mathbb{R}$ for all $i \in\{-1,0, \ldots, 2 k-1\}$, such that

$$
\begin{aligned}
& \Omega_{j}(t)=\sum_{i=1}^{2 k-1} c_{i, j}(q, T) \mu_{i}(t) \text { and } \\
& \Omega_{k+1}(t)=\sum_{j=0}^{2 k-1} g_{j}(q, T) \mu_{j}(t)+g_{-1}(q, T) \mu_{0}(t-T)
\end{aligned}
$$

hold for all $t \geq 0$.

Lemma 1 follows by first using the Binomial Theorem to write $Q(t, \ell, \ell+T)$ as a linear combination of the terms $(t-\ell)^{r}$ with $k-1 \leq r \leq 2 k-2$. In the next lemma (which can be deduced from [15] on linear systems), we say that a linear system is not exponentially unstable provided its poles are all in the closed left-half plane:

Lemma 2: Let $k \geq 2$ be an integer and $v=\left(v_{1}, \ldots, v_{k}\right)$ be any vector of $k$ real constants such that

$$
\left\{\begin{aligned}
\dot{z}_{i}(t) & =z_{i+1}(t), \quad i \in\{1, \ldots, k-1\} \\
\dot{z}_{k}(t) & =u+\sum_{i=1}^{k} v_{i} z_{i}
\end{aligned}\right.
$$

is not exponentially unstable when $u=0$. Then there is a bounded locally Lipschitz function $\vartheta$ such that (4), in closed loop with $u=\vartheta(Z)$ where $Z=\left(z_{1}, \ldots, z_{k}\right)^{\top}$, is globally asymptotically and locally exponentially stable to 0 .

In addition to the notation from the preceding two lemmas, our theorem will also use the nonzero constant

$$
b_{T}=\int_{-T}^{0} e^{q \ell} \ell^{k-1}(\ell+T)^{k-1} \mathrm{~d} \ell
$$

and the following assumption:

Assumption 1: (i) The function $\mathcal{F}$ in (1) is continuous in $t$ and globally Lipschitz in $\left(x, z_{1}\right)$ and satisfies $\mathcal{F}(t, 0,0)=0$ for all $t \geq 0$. (ii) There is a globally Lipschitz bounded function $\bar{\omega}: \mathbb{R}^{n} \rightarrow[-\bar{\omega}, \bar{\omega}]$ having some constant bound $\bar{\omega}>0$ such that $\omega(0)=0$, and constants $T>0$ and $q>0$, such that for each continuous function $\delta:[0,+\infty) \rightarrow \mathbb{R}$ that exponentially converges to 0 , the following is true: All solutions $\xi:[0,+\infty) \rightarrow \mathbb{R}^{n}$ of the system

$$
\begin{aligned}
& \dot{\xi}(t)= \\
& \mathcal{F}\left(t, \xi(t), \int_{t-T}^{t} \frac{e^{q(\ell-t)} Q(t, \ell, \ell+T) \omega(\xi(\ell))}{b_{T}} \mathrm{~d} \ell+\delta(t)\right)
\end{aligned}
$$

satisfy $\lim _{t \rightarrow+\infty} \xi(t)=0$, where $Q(t, a, b)=(t-a)^{k-1}(t-$ $b)^{k-1}$ and $k \geq 2$ is from (1).

We refer to part (ii) of Assumption 1 as our converginginput-converging-state assumption; see Section III for sufficient conditions. The system (6) differs from the nonlinear subsystem of (1) because the third argument of $\mathcal{F}$ in (1) has been replaced by the sum of $\delta(t)$ and an integral. In terms of the Jordan matrix

$J_{2 k-1}=\left[\begin{array}{ccccc}-q & 1 & 0 & \cdots & 0 \\ 0 & -q & 1 & \cdots & 0 \\ \vdots & \ddots & \ddots & \ddots & \vdots \\ \vdots & & \ddots & -q & 1 \\ 0 & \cdots & \cdots & 0 & -q\end{array}\right] \in \mathbb{R}^{(2 k-1) \times(2 k-1)}$

our main result is as follows, where the forward completeness of the closed loop system follows from the boundedness of $\omega$ and the control and the global Lipschitzness of $\mathcal{F}$ and $\omega$ :

Theorem 1: Let the constants $q>0, k \geq 2$, and $T>$ 0 and the functions $\mathcal{F}$ and $\omega$ be such that Assumption 1 holds, where $k \in \mathbb{N}$. Let $\vartheta$ and $v$ satisfy the requirements from Lemma 2. Consider the augmented $(x, Z, Y)$ system, consisting of (1) and the $2 k-1$ dimensional system

$$
\dot{Y}(t)=J_{2 k-1} Y(t)+\frac{e_{2 k-1}}{T} \frac{\omega(x(t))}{b_{T}}
$$

where $e_{2 k-1}=(0,0, \ldots, 1)^{\top} \in \mathbb{R}^{2 k-1}$ is the $(2 k-1)$-st standard basis vector, in closed loop with the control

$$
\begin{aligned}
& u\left(Z(t), Y_{t}, x_{t}\right)=\sigma_{\bar{a}}\left(\mathcal{M}\left(Y_{t}\right)\right) \\
& +g_{0}(q, T) \frac{\omega(x(t))}{b_{T}}+g_{-1}(q, T) \frac{\omega(x(t-T))}{b_{T}}+\vartheta\left(Z_{\star}(t)\right)
\end{aligned}
$$

with the saturation level

$$
\bar{a}=\left|\sum_{j=1}^{k} v_{j} \mathcal{C}_{j}(q, T)-\mathcal{G}(q, T)\right| e^{\left|J_{2 k-1}\right| T} \frac{\bar{\omega}}{b_{T}},
$$

where

$$
\mathcal{M}\left(Y_{t}\right)=\left(\mathcal{G}(q, T)-\sum_{j=1}^{k} v_{j} \mathcal{C}_{j}(q, T)\right) \Psi\left(Y_{t}\right)
$$

and $\Psi\left(Y_{t}\right)=Y(t)-e^{T J_{2 k-1}} Y(t-T)$ and

$$
Z_{\star}(t)=\left[\begin{array}{c}
z_{1}(t)-\mathcal{C}_{1}(q, T) \Psi\left(Y_{t}\right) \\
\vdots \\
z_{k}(t)-\mathcal{C}_{k}(q, T) \Psi\left(Y_{t}\right)
\end{array}\right],
$$

and $\mathcal{G}(q, T)=\left[g_{2 k-1}(q, T) \ldots \ldots g_{1}(q, T)\right]$ and

$$
\mathcal{C}_{j}(q, T)=\left[c_{2 k-1, j}(q, T) \ldots \ldots c_{1, j}(q, T)\right], 1 \leq j \leq k
$$

and where the constants $c_{i, j}$ and $g_{i}$ satisfy the requirements from Lemma 1 for the function $\mu_{0}(t)=\omega(x(t)) / b_{T}$. Then all maximal solutions $(x, Z, Y)(t)$ of the augmented $(x, Z, Y)$ system satisfy $\lim _{t \rightarrow+\infty}(x, Z, Y)(t)=0$.

We next provide sufficient conditions for our converginginput-converging-state condition from Assumption 1 to hold, and then we prove Theorem 1 in Section IV.

\section{Checking Assumption 1}

We provide sufficient conditions for our converging-inputconverging-state conditions on (6) to hold, based on Lyapunov functions, using

$$
\dot{q}(t)=\mathcal{F}(t, q(t), \omega(q(t))),
$$


where $\mathcal{F}$ is from (1), and using the standard definitions of positive definiteness, properness, and $\mathcal{K}_{\infty}$ [6]. In the next assumption, $V_{t}$ and $V_{x}$ are the partial derivative with respect to $t$ and the gradient with respect to $x$, respectively, and uniform global Lipschitzness in $x$ means that the global Lipschitz constants can be chosen independently of $t$ :

Assumption 2: There exist functions $f:[0,+\infty) \times \mathbb{R}^{n} \rightarrow$ $\mathbb{R}^{n}$ and $g:[0,+\infty) \times \mathbb{R}^{n} \rightarrow \mathbb{R}^{n}$ that are uniformly globally Lipschitz in $x$ and continuous on $[0,+\infty) \times \mathbb{R}^{n}$, such that

$$
\mathcal{F}(t, x, p)=f(t, x)+g(t, x) p
$$

holds for all $t \geq 0, x \in \mathbb{R}^{n}$, and $p \in \mathbb{R}$. Also, there exist a $C^{1}$ uniformly proper and positive definite function $V$ : $[0,+\infty) \times \mathbb{R}^{n} \rightarrow[0,+\infty)$; a uniformly continuous positive definite function $W: \mathbb{R}^{n} \rightarrow[0,+\infty)$; positive constants $r_{0}$, $r_{1}$, and $r_{3}$; and a constant $r_{2} \geq 0$ such that for all $(t, x) \in$ $[0,+\infty) \times \mathbb{R}^{n}$, we have

$$
\begin{aligned}
& V_{t}(t, x)+V_{x}(t, x)(f(t, x)+g(t, x) \omega(x)) \leq-W(x), \\
& \left|V_{x}(t, x) g(t, x)\right| \leq r_{0} \sqrt{W(x)},|\omega(x)| \leq r_{1} \sqrt{W(x)}, \\
& |f(t, x)| \leq r_{2} \sqrt{W(x)}, \text { and }|g(t, x)| \leq r_{3},
\end{aligned}
$$

where $\omega: \mathbb{R}^{n} \rightarrow \mathbb{R}$ is bounded and satisfies $\omega(0)=0$, and there is a global Lipschitz constant $C>0$ for $\omega$ on $\mathbb{R}^{n}$.

See [11] for conditions under which Assumption 2 holds.

Proposition 1: If Assumption 2 holds, then for all constants $T>0$ such that

$$
4\left(T r_{0} C\right)^{2}\left[2 r_{2}^{2}+\frac{5}{2}\left(\frac{r_{1} r_{3} T^{2 k-1}}{\left|b_{T}\right|}\right)^{2}\right]<1,
$$

and for all integers $k \geq 2$, Assumption 1 is satisfied.

Proof: (Sketch.) The proof has a similar structure to the proof of the sufficient conditions from [10], so we only sketch the proof. Fix any continuous function $\delta:[0,+\infty) \rightarrow$ $\mathbb{R}$ that exponentially converges to 0 . By (5), we have

$$
\int_{t-T}^{t} \frac{e^{q(\ell-t)} Q(t, \ell, \ell+T)}{b_{T}} \mathrm{~d} \ell=1
$$

for all $t \geq T$. Hence, along all solutions $x(t)$ of (6), we get

$$
\begin{aligned}
& \dot{V}(t) \leq-W(x(t))+r_{0} \sqrt{W(x(t))} \\
& \times\left(\sup _{\ell \in[t-T, t]}|\omega(x(\ell))-\omega(x(t))|+|\delta(t)|\right)
\end{aligned}
$$

for all $t \geq T$ and

$$
\begin{aligned}
& |\dot{x}(t)| \leq r_{2} \sqrt{W(x(t))}+ \\
& r_{3}\left(\frac{T^{2(k-1)}}{\left|b_{T}\right|} \int_{t-T}^{t}|\omega(x(\ell))| \mathrm{d} \ell+|\delta(t)|\right)
\end{aligned}
$$

along all solutions of (6). It follows from the Young and Jensen's inequalities that along all solutions of (6), we have

$$
\begin{aligned}
& |\dot{x}(t)|^{2} \leq 2 r_{2}^{2} W(x(t))+ \\
& 2 r_{3}^{2}\left(5|\delta(t)|^{2}+\frac{5}{4} \frac{T^{4(k-1)} T r_{1}^{2}}{b_{T}^{2}} \int_{t-T}^{t} W(x(\ell)) \mathrm{d} \ell\right) .
\end{aligned}
$$

Hence, Jensen's and Young's inequalities also give

$$
\begin{aligned}
\dot{V}(t) \leq & -\frac{1}{2} W(x(t))+r_{0}^{2}\left(|\delta(t)|^{2}+\right. \\
& \left.C^{2} \sup _{\ell \in[t-T, t]}|x(\ell)-x(t)|^{2}\right) \\
\leq & -\frac{1}{2} W(x(t))+r_{0}^{2}\left(|\delta(t)|^{2}\right. \\
& \left.+C^{2} T \int_{t-T}^{t}|\dot{x}(\ell)|^{2} \mathrm{~d} \ell\right) \\
\leq & -\frac{1}{2} W(x(t))+r_{0}^{2}\left(|\delta(t)|^{2}\right. \\
& +C^{2} T\left[2 r_{2}^{2} \int_{t-T}^{t} W(x(\ell)) \mathrm{d} \ell\right. \\
& +10 r_{3}^{2} T|\delta|_{[t-T, t]}^{2} \\
& \left.\left.+\frac{5}{2} r_{3}^{2} \frac{T^{4(k-1)} T^{2}}{b_{T}^{2}} r_{1}^{2} \int_{t-2 T}^{t} W(x(\ell)) \mathrm{d} \ell\right]\right) \\
\leq & -\frac{1}{2} W(x(t))+\mathcal{N}_{1} \int_{t-2 T}^{t} W(x(\ell)) \mathrm{d} \ell \\
& +\mathcal{N}_{2}|\delta|_{[t-T, t]}^{2}
\end{aligned}
$$

along all solutions of (6) for all $t \geq T$, where

$$
\mathcal{N}_{1}=T\left(r_{0} C\right)^{2}\left(2 r_{2}^{2}+\frac{5}{2}\left(\frac{r_{1} r_{3} T^{2 k-1}}{\left|b_{T}\right|}\right)^{2}\right)
$$

and $\mathcal{N}_{2}=10\left(r_{0} r_{3} T C\right)^{2}+r_{0}^{2}$. Then our condition (16) provides a constant $\lambda>1$ such that $2 T \mathcal{N}_{1} \lambda<1 / 2$, so

$$
V_{1}\left(t, x_{t}\right)=V(t, x(t))+\lambda \mathcal{N}_{1} \int_{t-2 T}^{t} \int_{s}^{t} W(x(\ell)) \mathrm{d} \ell \mathrm{d} s
$$

satisfies the following along all solutions of (6):

$$
\dot{V}_{1} \leq-\left\{\frac{1}{2}-2 T \mathcal{N}_{1} \lambda\right\} W(x(t))+\mathcal{N}_{2}|\delta|_{[t-T, t]}^{2}
$$

for all $t \geq 2 T$. The result now follows from our choice of $\lambda$ and applying Barbalat's Lemma to $W(x(t))$.

\section{PROOF OF THEOREM 1}

Theorem 1 will follow from three more lemmas, which we state next. The following follows from [14, Appendix A.3]:

Lemma 3: For the Jordan matrix $J_{2 k-1}$, the equality

$$
e^{J_{2 k-1} t}=e^{-q t}\left[\begin{array}{ccccc}
1 & t & \frac{t^{2}}{2} & \ldots & \frac{t^{2(k-1)}}{(2(k-1)) !} \\
0 & 1 & t & \ldots & \frac{t^{2 k-3}}{(2 k-3) !} \\
\vdots & \ddots & \ddots & \ddots & \vdots \\
\vdots & & \ddots & 1 & t \\
0 & \ldots & \ldots & 0 & 1
\end{array}\right]
$$

holds for all $t \in \mathbb{R}$ and integers $k \geq 2$.

Lemma 4: Let $\mu_{0}:[-T,+\infty) \rightarrow[-\bar{\mu}, \bar{\mu}]$ be any continuous function having a bound $\bar{\mu}$. Then the functions $\mu_{i}$ from (2) in Lemma 1, and the functions $\Psi\left(Y_{t}\right)=Y(t)-$ $e^{T J_{2 k-1}} Y(t-T)$ for all solutions $Y$ of

$$
\dot{Y}(t)=J_{2 k-1} Y(t)+\frac{e_{2 k-1}}{T} \mu_{0}(t),
$$

are such that for all $t \geq T$, we have

$$
\nu_{2 k-1}(t)=\Psi\left(Y_{t}\right) \text { and }\left|\Psi\left(Y_{t}\right)\right| \leq e^{\left|J_{2 k-1}\right| T} \bar{\mu},
$$

where $\nu_{2 k-1}(t)=\left(\mu_{2 k-1}(t), \ldots, \mu_{1}(t)\right)^{\top}$ for all $t \geq T$. 
Proof: By integrating (26), we deduce that

$$
\Psi\left(Y_{t}\right)=Y(t)-e^{T J_{2 k-1}} Y(t-T)=\varrho(t),
$$

where $\varrho(t)=\int_{t-T}^{t} e^{J_{2 k-1}(t-\ell)} \frac{e_{2 k-1}}{T} \mu_{0}(\ell) \mathrm{d} \ell$

for all $t \geq T$. On the other hand, using (25), we obtain

$$
\varrho(t)=
$$

which proves the first conclusion of the lemma. The second conclusion of the lemma follows from (28)-(29).

Lemma 5: Let $\mu_{0}:[-T,+\infty) \rightarrow[-\bar{\mu}, \bar{\mu}]$ be any continuous function having a bound $\bar{\mu}$, and let the constants $v_{i}$ and the function $\vartheta$ satisfy the requirements from Lemma 2 . Consider the linear system

$$
\left\{\begin{aligned}
\dot{z}_{i}(t) & =z_{i+1}(t), \quad i \in\{1, \ldots, k-1\} \\
\dot{z}_{k}(t) & =u(t)+\sum_{j=1}^{k} v_{j} z_{j}(t)
\end{aligned}\right.
$$

in closed loop with the control

$$
\begin{aligned}
& u\left(Z(t), Y_{t}, x_{t}\right)=\sigma_{\bar{a}}\left(\mathcal{M}\left(Y_{t}\right)\right) \\
& +g_{0}(q, T) \mu_{0}(t)+g_{-1}(q, T) \mu_{0}(t-T)+\vartheta\left(Z_{\star}(t)\right)
\end{aligned}
$$

with the saturation level $\bar{a}$ for $\sigma_{\bar{a}}$ defined by

$$
\bar{a}=\left|\sum_{j=1}^{k} v_{j} \mathcal{C}_{j}(q, T)-\mathcal{G}(q, T)\right| e^{\left|J_{2 k-1}\right| T} \bar{\mu}
$$

and where $Y$ satisfies (26) and $\mathcal{M}, \Psi, Z_{*}, \mathcal{G}$, and the $\mathcal{C}_{j}$ 's and $g_{j}$ 's are defined as in Theorem 1 . Then the dynamics for the vector $\tilde{Z}(t)=\left(\tilde{z}_{1}(t), \ldots, \tilde{z}_{k}(t)\right)$ are globally asymptotically and locally exponentially stable to the origin, where $\tilde{z}_{i}(t)=$ $z_{i}(t)-\Omega_{i}(t)$ for $i=1,2, \ldots, k$ and the $\Omega_{i}$ 's are defined in (2) in Lemma 1.

Proof: The fact that $\dot{\Omega}_{i}=\Omega_{i+1}$ for all $i \in\{1,2, \ldots, k\}$ and the structure of the dynamics (31) allow us to conclude that the dynamics for the functions $\tilde{z}_{i}(t)=z_{i}(t)-\Omega_{i}(t)$ are

$$
\left\{\begin{aligned}
\dot{\tilde{z}}_{i}(t)= & \tilde{z}_{i+1}(t), \quad i \in\{1, \ldots, k-1\} \\
\dot{\tilde{z}}_{k}(t)= & u(t)-\Omega_{k+1}(t) \\
& +\sum_{j=1}^{k} v_{j}\left[\tilde{z}_{j}(t)+\Omega_{j}(t)\right]
\end{aligned}\right.
$$

Using our conclusion from Lemma 4 that

$$
\nu_{2 k-1}(t)=\Psi\left(Y_{t}\right)
$$

where $\nu_{2 k-1}(t)=\left(\mu_{2 k-1}(t), \ldots, \mu_{1}(t)\right)^{\top}$ as before, it follows from (3) that

$$
\begin{aligned}
\dot{\tilde{z}}_{k}(t)= & u(t)-\mathcal{G}(q, T) \nu_{2 k-1}(t)-g_{0}(q, T) \mu_{0}(t) \\
& -g_{-1}(q, T) \mu_{0}(t-T) \\
& +\sum_{j=1}^{k} v_{j} \tilde{z}_{j}(t)+\sum_{j=1}^{k} v_{j} \mathcal{C}_{j}(q, T) \nu_{2 k-1} .
\end{aligned}
$$

Hence (35) and our choice

$$
\dot{Y}(t)=J_{2 k-1} Y(t)+\frac{e_{2 k-1}}{T} \mu_{0}(t)
$$

of our dynamic extension imply that for all $t \geq T$, we have

$$
\left\{\begin{aligned}
\dot{\tilde{z}}_{i}(t)= & \tilde{z}_{i+1}(t), \quad i \in\{1, \ldots, k-1\} \\
\dot{\tilde{z}}_{k}(t)= & u(t)+\sum_{j=1}^{k} v_{j} \tilde{z}_{j}(t)-g_{0}(q, T) \mu_{0}(t) \\
& -g_{-1}(q, T) \mu_{0}(t-T)+\bar{g} \Psi\left(Y_{t}\right)
\end{aligned}\right.
$$

where

$$
\bar{g}=\sum_{j=1}^{k} v_{j} \mathcal{C}_{j}(q, T)-\mathcal{G}(q, T) .
$$

Next note that since Lemma 1 gives

$$
\Omega_{j}=\mathcal{C}_{j}(q, T) \nu_{2 k-1} \text { for } 1 \leq j \leq k,
$$

it follows that for all $i \in\{1, \ldots, k\}$, we have

$$
\tilde{z}_{i}(t)=z_{i}(t)-\Omega_{i}(t)=z_{i}(t)-\mathcal{C}_{i}(q, T) \nu_{2 k-1}(t) .
$$

Thus, (35) gives $\tilde{z}_{i}(t)=z_{i}(t)-\mathcal{C}_{i}(q, T) \Psi\left(Y_{t}\right)$ for all $t \geq T$, so $Z_{\star}(t)=\tilde{Z}(t)$ for all $t \geq T$. Therefore, our choice (32) of the control gives

$$
\left\{\begin{aligned}
\dot{\tilde{z}}_{i}(t)= & \tilde{z}_{i+1}(t), \quad i \in\{1, \ldots, k-1\} \\
\dot{\tilde{z}}_{k}(t)= & \sum_{j=1}^{k} v_{j} \tilde{z}_{j}(t)+\sigma_{\bar{a}}\left(-\bar{g} \Psi\left(Y_{t}\right)\right) \\
& +\bar{g} \Psi\left(Y_{t}\right)+\vartheta\left(Z_{\star}(t)\right) .
\end{aligned}\right.
$$

According to (27), we have $\left|\bar{g} \Psi\left(Y_{t}\right)\right| \leq|\bar{g}| e^{\left|J_{2 k-1}\right| T} \bar{\mu}$ for all $t \geq T$. From the definition of $\sigma_{\bar{a}}$, it follows that for all $t \geq T$, we have

$$
\left\{\begin{array}{l}
\dot{\tilde{z}}_{i}(t)=\tilde{z}_{i+1}(t), \quad i \in\{1, \ldots, k-1\} \\
\dot{\tilde{z}}_{k}(t)=\vartheta(\tilde{Z}(t))+\sum_{i=1}^{k} v_{i} \tilde{z}_{i}
\end{array}\right.
$$

so the lemma follows from our choice of $\vartheta$ in Lemma 2.

We now combine the preceding lemmas to prove Theorem 1. First notice that the closed loop system is as follows:

$$
\left\{\begin{aligned}
\dot{x}(t) & =\mathcal{F}\left(t, x(t), z_{1}(t)\right) \\
\dot{z}_{i}(t) & =z_{i+1}(t), \quad i \in\{1, \ldots, k-1\} \\
\dot{z}_{k}(t) & =u\left(Z(t), Y_{t}, x_{t}\right)+\sum_{j=1}^{k} v_{j} z_{j}(t) \\
\dot{Y}(t) & =J_{2 k-1} Y(t)+\frac{e_{2 k-1}}{T} \frac{\omega(x(t))}{b_{T}}
\end{aligned}\right.
$$

which is forward complete by our global Lipschitzness assumptions. Using the preceding lemma, we deduce that

$$
\lim _{t \rightarrow+\infty}\left|z_{i}(t)-\Omega_{i}(t)\right|=0
$$

for all $i=1$ to $k$, and $z_{1}-\Omega_{1}$ exponentially converges to 0 .

Next notice that $\zeta=\Omega_{1}$ in Lemma 1 , and therefore that

$$
\dot{x}(t)=\mathcal{F}\left(t, x(t), \zeta(t)+z_{1}(t)-\Omega_{1}(t)\right)
$$

when we choose the bounded function $\mu_{0}(t)=\omega(x(t)) / b_{T}$ 
to obtain

$$
\zeta(t)=\int_{t-T}^{t} e^{q(\ell-t)} Q_{1}(t, \ell, \ell+T) \frac{\omega(x(\ell))}{b_{T}} d \ell .
$$

Hence, we can use Assumption 1 with $\delta=z_{1}-\Omega_{1}$ to conclude that

$$
\lim _{t \rightarrow+\infty}|x(t)|=0
$$

and therefore that for all $i \in\{1,2, \ldots, k\}$, we have

$$
\lim _{t \rightarrow+\infty} \Omega_{i}(t)=0,
$$

since $\omega(0)=0$ and $\omega$ is continuous at 0 . Therefore, Theorem 1 follows by combining (45) with (48)-(49).

\section{ILLUSTRATION}

We apply our method to the three-dimensional dynamics

$$
\left\{\begin{aligned}
\dot{x}(t) & =\frac{|x(t)|}{1+|x(t)|}+z_{1}(t) \\
\dot{z}_{1}(t) & =z_{2}(t) \\
\dot{z}_{2}(t) & =u(t)
\end{aligned}\right.
$$

which is outside the scope of classical backstepping, as the right side of $\dot{x}(t)$ is not differentiable. With our earlier notation, we choose $k=2, n=1, q=1$, and

$$
\omega(x)=-\frac{|x|}{1+|x|}-2 \frac{x}{1+|x|} .
$$

We find a constant $T>0$ such that Assumption 1 holds with

$$
\mathcal{F}\left(t, x, z_{1}\right)=\frac{|x|}{1+|x|}+z_{1} .
$$

Since (51)-(52) are globally Lipschitz functions in the states and $\mathcal{F}$ is affine in $z_{1}$ and $\omega$ is bounded, it suffices to produce constants $r_{i}$ for $i=0,1,2,3$ and functions $V$ and $W$ that satisfy Assumption 2 with $f(t, x)=x /(1+|x|)$ and $g(t, x)=1$, and then we choose $T$ such that (16) holds.

To this end, we check that Assumption 2 holds for

$$
V(t, x)=\int_{0}^{x} \sigma_{1}(\ell) \mathrm{d} \ell \text { and } W(x)=\frac{2 \sigma_{1}(x) x}{1+|x|} .
$$

We have

$$
f(t, x)+g(t, x) \omega(x)=-\frac{2 x}{1+|x|}
$$

so our conditions on the $r_{i}$ 's from (15) will hold if

$$
\begin{aligned}
& \left|\sigma_{1}(x)\right| \leq r_{0} \sqrt{\frac{2 \sigma_{1}(x) x}{1+|x|}}, \quad \frac{3|x|}{1+|x|} \leq r_{1} \sqrt{\frac{2 \sigma_{1}(x) x}{1+|x|}}, \\
& \frac{|x|}{1+|x|} \leq r_{2} \sqrt{\frac{2 \sigma_{1}(x) x}{1+|x|}}, \text { and } 1 \leq r_{3} .
\end{aligned}
$$

By separately considering points where $x \in[-1,1]$ or $x \notin[-1,1]$, we can readily check that Assumption 2 is satisfied using $C=3, r_{0}=1, r_{1}=\frac{3}{\sqrt{2}}, r_{2}=1$, and $r_{3}=1$. Moreover, for the case where $k=2$ and $q=1$, our constant $b_{T}$ from (5) that is used in our condition (16) on $T$ in Proposition 1 is $b_{T}=2-T-e^{-T}(2+T)$, so our requirement (16) on $T>0$ is that

$$
\begin{aligned}
& 1>4\left(T r_{0} C\right)^{2}\left[2 r_{2}^{2}+\frac{5}{2}\left(\frac{r_{1} r_{3} T^{3}}{\left|b_{T}\right|}\right)^{2}\right] \\
& =4(3 T)^{2}\left[2+\frac{5}{2}\left(\frac{3 T^{3}}{\sqrt{2}\left|2-T-e^{-T}(2+T)\right|}\right)^{2}\right]
\end{aligned}
$$

and we can use Mathematica [18] to check that the right side of (56) is 0.912536 for $T=0.11$. Hence, Assumption 1 holds with $T=0.11$, so the desired dynamic controller is provided by Theorem 1 .

\section{CONCLUSIONS}

Our new bounded backstepping technique applies to a large class of partially linear systems with arbitrarily large numbers of integrators, when the nonlinear subsystems satisfy a converging-input-converging-state assumption. For many cases, our Lyapunov functions provide sufficient conditions for our converging-input-converging-state assumption to hold. Our theorem provides bounded controllers for the original system. We plan to combine our methods with the time delay methods in [4] to also allow measurement delays.

\section{REFERENCES}

[1] B. Bialy, J. Klotz, J. Curtis, and W. Dixon. Adaptive backstepping controller for a hypersonic air-breathing missile. In Proc. AIAA Guidance Navigation and Control Conference, Minneapolis, MN, Paper AIAA 2012-4468, 2012.

[2] W. Dixon, E. Zergeroglu, D. Dawson, and M. Hannan. Global adaptive partial state feedback tracking control of rigid-link flexiblejoint robots. Robotica, 18:325-336, 2000.

[3] K. Dupree, C-H. Liang, G. Hu, and W. Dixon. Adaptive Lyapunovbased control of a robot and mass-spring system undergoing an impact collision. IEEE Transactions on Systems, Man, and Cybernetics Part B: Cybernetics, 38(4):1050-1061, 2008.

[4] M. Krstic. Delay Compensation for Nonlinear, Adaptive, and PDE Systems. Birkhauser, Boston, 2009.

[5] M. Krstic, I. Kanellakopoulos, and P. Kokotovic. Nonlinear and Adaptive Control Design. John Wiley and Sons, New York, NY, 1995.

[6] M. Malisoff and F. Mazenc. Constructions of Strict Lyapunov Functions. Springer-Verlag, London, 2009.

[7] F. Mazenc and S. Bowong. Backstepping with bounded feedbacks for time varying systems. SIAM Journal on Control and Optimization, 43(3):856-871, 2004

[8] F. Mazenc and A. Iggidr. Backstepping with bounded feedbacks. Systems and Control Letters, 51(3-4):235-245, 2004.

[9] F. Mazenc and M. Malisoff. Bounded backstepping approach under input delays. In Proc. European Control Conference, pages 20562061, Linz, Austria, 2015.

[10] F. Mazenc and M. Malisoff. New control design for bounded backstepping under input delays. Automatica, 66:48-55, 2016.

[11] F. Mazenc, M. Malisoff, and Z. Lin. Further results on input-to-state stability for nonlinear systems with delayed feedbacks. Automatica, 44(9):2415-2421, 2008.

[12] F. Mazenc, M. Malisoff, and J. Weston. New bounded backstepping control designs for time-varying systems under converging input converging state conditions. In Proc. IEEE Conference on Decision and Control, pages 3167-3171, Las Vegas, NV, USA, 2016.

[13] F. Mazenc, S-I. Niculescu, and M. Bekaik. Backstepping for nonlinear systems with delay in the input revisited. SIAM Journal on Control and Optimization, 49(6):2263-2278, 2011.

[14] E. Sontag. Mathematical Control Theory: Deterministic FiniteDimensional Systems. Springer, New York, NY, 1998.

[15] H. Sussmann, E. Sontag, and Y. Yang. A general result on the stabilization of linear systems using bounded controls. IEEE Transactions on Automatic Control, 39(12):2411-2425, 1994.

[16] J. Tsinias. Input to state stability properties of nonlinear systems and applications to bounded feedback stabilization using saturation. ESAIM Control, Optimization, and Calculus of Variations, 2:57-87, 1997.

[17] J. Tsinias. Backstepping design for time-varying systems and application to partial-static feedback and asymptotic tracking. Systems and Control Letters, 39:219-227, 2000.

[18] Wolfram Mathematica. The world's definitive system for modern technical computing, 2015. Wolfram Research, http://www.wolfram.com/mathematica/, Accessed September 20, 2017. 